\title{
Serum biomarkers combined with uterine artery Doppler in prediction of preeclampsia
}

\author{
LIJIE LI ${ }^{1}$, YANMEI ZHENG ${ }^{1}$, YING ZHU ${ }^{1}$ and JIANCHUN LI ${ }^{2}$ \\ Departments of ${ }^{1}$ Obstetrics and ${ }^{2}$ Ultrasonic Diagnosis, Zhejiang Provincial People's Hospital, \\ Hangzhou, Zhejiang 310014, P.R. China
}

Received May 18, 2016; Accepted August 18, 2016

DOI: 10.3892/etm.2016.3625

\begin{abstract}
First-trimester screening may be a major advantage over a second-trimester approach since it opens prospects for early and more efficient interventions. The aim of the current study was to evaluate whether the measurement of maternal serum inhibin A, activin A and placental growth factor (PlGF) at three to four months' gestation with the second-trimester uterine artery pulsatility index (PI) are useful in predicting preeclampsia in a group of nulliparous women. All the patients also underwent uterine artery Doppler examination to measure the PI at 22-24 weeks' gestation. Inhibin A, activin A and PlGF were measured using an ELISA by an examiner who was blinded to the pregnancy outcome. Thirty-eight cases with preeclampsia and 100 controls were analyzed. Second-trimester uterine artery PI and marker levels were expressed as multiples of the median (MoM). The uterine artery PI was increased in pregnancies with preeclampsia compared with controls. In pregnancies that developed preeclampsia, the uterine artery PI was increased $(1.61 \pm 0.047$ vs. $1.02 \pm 0.049, \mathrm{P}<0.001)$, as was the level of inhibin A $(1.72 \pm 0.023$ vs. $1.03 \pm 0.063, \mathrm{P}<0.001)$ and the level of activin A $(1.68 \pm 0.38$ vs. $1.06 \pm 0.42, \mathrm{P}<0.001)$ compared with the controls. In contrast, the level of PIGF was decreased in pregnancies that developed preeclampsia compared with the controls $(0.69 \pm 0.23$ vs. $1.00 \pm 0.26, \mathrm{P}<0.001)$. A combination of activin A, PlGF and uterine artery PI gave an AUC of 0.915 (95\% CI, 0.812-0.928; $\mathrm{P}<0.001)$ with a sensitivity of $91 \%$ at a specificity of $82 \%$. In our study, we demonstrated that both serum inhibin A and activin A levels were increased, while the PIGF level was decreased in the early second-trimester in women who developed preeclampsia.
\end{abstract}

\section{Introduction}

Preeclampsia is a pregnancy-associated disorder, characterized by hypertension and proteinuria. It affects approximately

Correspondence to: Dr Jianchun Li, Department of Ultrasonic Diagnosis, Zhejiang Provincial People's Hospital, 158 Shangtang Road, Hangzhou, Zhejiang 310014, P.R. China

E-mail: jian_chunli1@163.com

Key words: preeclampsia, inhibin A, activin A, prediction
$3 \%$ of pregnant women, and contributes substantially to maternal and fetal morbidity and mortality worldwide $(1,2)$. Pathophysiology of the disease mainly occurs in early pregnancy due to a variety of immune and genetic factors, placental ischemia, oxidative stress and other factors resulting in vascular anomalies and placental site trophoblastic cell dysfunction, shallow implantation of the placenta, and increased resistance of uterine artery blood flow $(3,4)$. This leads to placental hypoperfusion and functional decline, and hypoxic-ischemic placental oxidative stress occurs locally, producing a variety of active polypeptide substances into the maternal circulation, causing maternal systemic small artery spasm (5). In China, the incidence of preeclampsia ranges from 9.4 to $10.4 \%$ and it seriously affects preterm labor and fetal growth restriction (FGR) (6). Inadequate trophoblast invasion of the maternal spiral arteries during early gestation is thought to contribute to its etiology.

Currently, women at risk are mainly identified based on clinical history. Nulliparity is one of the major clinical risk factors for the development of preeclampsia. Other relevant factors include an increased body mass index and other medical conditions such as prepregnancy diabetes, previous preeclampsia, or chronic hypertension $(7,8)$. However, screening by maternal history alone detects only $30 \%$ of women who may develop preeclampsia. The clinical risk-based strategy is not effective for nulliparous women without other risk factors. With increased understanding of the pathogenesis of preeclampsia, clinical prediction models of the disease have gradually diversified (9). Doppler has more recently shown some promise. Indeed, Campbell first introduced color Doppler ultrasound to investigate the uteroplacental circulation. Uterine artery pulsatility index (PI) has been used as a marker of preeclampsia and FGR, in the presence of which PI increases due to the elevation in uterine artery impedance (10).

Although much research into the mechanism of preeclampsia has been carried out, its exact pathogenesis remains poorly known. Recently, studies have found that preeclampsia is associated with a decreased level of serum placental growth factor (PlGF), as well as increased levels of inhibin A and activin A $(11,12)$, although a systematic review of screening tests for preeclampsia have concluded that no single test is yet available to provide good diagnostic accuracy $(7,13)$. A combined screening involving several relevant markers is more likely to provide the best prediction. 
Table I. Demographic characteristics of the women with preeclampsia in the study group and the normal controls.

\begin{tabular}{lccc}
\hline Characteristics & Cases (39) & Controls (100) & P-value \\
\hline Total no. & 39 & 100 & - \\
Primiparous & 38 & 95 & - \\
Pluriparous & 1 & 5 & - \\
Maternal age (years) & $27.12 \pm 3.12$ & $28.67 \pm 3.51$ & 0.06 \\
Maternal BMI $\left(\mathrm{kg} / \mathrm{m}^{2}\right)$ & $21.61 \pm 2.17$ & $21.43 \pm 2.52$ & 0.21 \\
GA at blood sample (days) & $101.01 \pm 6.23$ & $102.49 \pm 5.86$ & 0.73 \\
Systolic BP $(\mathrm{mmHg})$ & $151.03 \pm 8.13$ & $121.33 \pm 9.91$ & $<0.001$ \\
Diastolic BP (mmHg) & $98.17 \pm 4.34$ & $72.51 \pm 6.99$ & $<0.001$ \\
GA at delivery (weeks) & $35.12 \pm 2.34$ & $39.51 \pm 1.24$ & $<0.001$ \\
Birth weight (g) & $2,715.11 \pm 576.72$ & $3,243.51 \pm 324.21$ & $<0.001$ \\
\hline
\end{tabular}

BMI, body mass index; BP, blood pressure; GA, gestational age. Data are expressed as number or mean $\pm \mathrm{SD}$.

First-trimester screening would represent a major advantage over a second-trimester approach since it opens prospects for early and more efficient interventions $(14,15)$. Accordingly, we aimed at evaluating whether the measurement of maternal serum inhibin A, activin A and PlGF at 3-4 months' gestation or a combination of these biochemical markers with the second-trimester uterine artery PI are useful in predicting preeclampsia in a group of nulliparous women.

\section{Subjects and methods}

Ethics statement. The Medical Ethics Committee of the Zhejiang Provincial People's Hospital (Zhejiang, China) approved this study. Written informed consent conforming to the tenets of the Declaration of Helsinki were obtained from each participant prior to the study.

Participants. In this prospective cohort study, a total of 800 pregnant women at the time of screening for Down syndrome at 11-13 weeks, and 100 gestational age-matched normal pregnancies as controls were recruited at the Department of Urology, The First Hospital of Zhejiang Province, from January 2013 to January 2015. For cases in which no major fetal defect was detected, women were invited to undergo an additional screening study for preeclampsia and blood samples were taken, centrifuged to extract the serum and stored at $-80^{\circ} \mathrm{C}$ for subsequent analysis. Multiparous women, multiple gestations, and pregnancies with a major fetal chromosomal or structural anomaly were excluded from further analysis.

Doppler measurement. An ultrasound examination was carried out for diagnosis of major fetal defects, measurement of nuchal translucency thickness, and crown-rump length (CRL), which was used to determined gestational age. Furthermore, participants underwent Doppler measurement of the uterine artery PI at 22-24 weeks, performed by experienced sonographers who had obtained the Fetal Medicine Foundation's Certificate of Competence in Placental and Fetal Doppler. Uterine artery PI was calculated as the mean PI from three similar consecutive waveforms. All examinations were performed transabdominally using a C3-5-MHz curvilinear transducer (Envisor 2540A; Philips Medical Systems, Shenyang, China). The results of trimester Doppler were not communicated to the physicians in charge of the follow-up and were not recorded in the ultrasound report.

Diagnostic of preeclampsia. Preeclampsia was defined as systolic blood pressure $\geq 140 \mathrm{mmHg}$ and/or diastolic blood pressure $\geq 90 \mathrm{mmHg}$ on at least two occasions $4 \mathrm{~h}$ apart, developing after 20 weeks of gestation in previously normotensive women with proteinuria of $300 \mathrm{mg}$ or more in $24 \mathrm{~h}$, or two readings of at least ++ on dipstick analysis of midstream or catheter urine specimens if 24-h urine collection was not available.

Measurement of serum markers. Maternal nonfasting blood samples were collected, immediately centrifuged for $10 \mathrm{~min}$ at $3,800 \times \mathrm{g}$, divided into 4 aliquots, and frozen at $-80^{\circ} \mathrm{C}$ until used for the analyses. The samples were tested for inhibin A, activin $\mathrm{A}$ and PIGF levels using solid-phase sandwich enzyme linked immunosorbent assay (ELISA) (R\&D Systems, Minneapolis, MN, USA) by technicians who were blinded to the clinical outcome. According to the manufacturer, fresh quality-control samples were used at concentrations of 50 and $1000 \mathrm{pg} / \mathrm{ml}$ for PlGF, 50 and $1000 \mathrm{pg} / \mathrm{ml}$ for inhibin A, and 50 and $2500 \mathrm{pg} / \mathrm{ml}$ for activin A. The interassay coefficients of variation for the respective low and high concentrations were 5.64 and $7.96 \%$ for PIGF, 6.89 and $11.56 \%$ for inhibin A and 4.27 and $9 \%$ for activin A.

Statistical analysis. The inhibin A, activin A and PlGF results are expressed as multiples of the median (MoM). Basic demographic characteristics including weight and height as well as serum inhibin A, activin A and PlGF results in pregnancies with preeclampsia and normal pregnancies were compared by unpaired t-test. The sensitivity and specificity for different cut-offs of each variable in detecting preeclampsia were calculated and depicted as receiver operating characteristic (ROC) curves. Multiple logistic regression analysis was used to model the combination of inhibin A, activin A, PlGF and uterine 

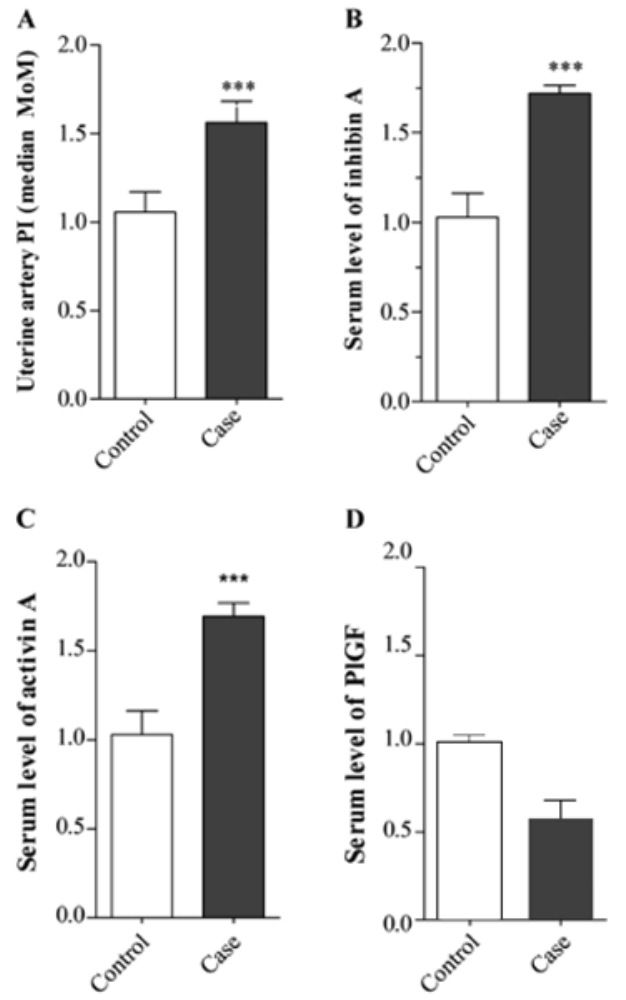

Figure 1. Measurement of uterine artery pulsatility index (PI) and serum markers. (A) Second-trimester uterine artery PI in 39 pregnancies which developed preeclampsia and in 100 normal controls. (B) Second-trimester inhibin A levels in 39 pregnancies which went on to develop preeclampsia and in 100 normal controls. (C) Second-trimester activin A levels in 39 pregnancies which went on to develop preeclampsia and in 100 normal controls. (D) Placental growth factor (PlGF) levels in 39 pregnancies which went on to develop preeclampsia and in 100 normal controls $\left({ }^{* * *} \mathrm{P}<0.001\right)$.

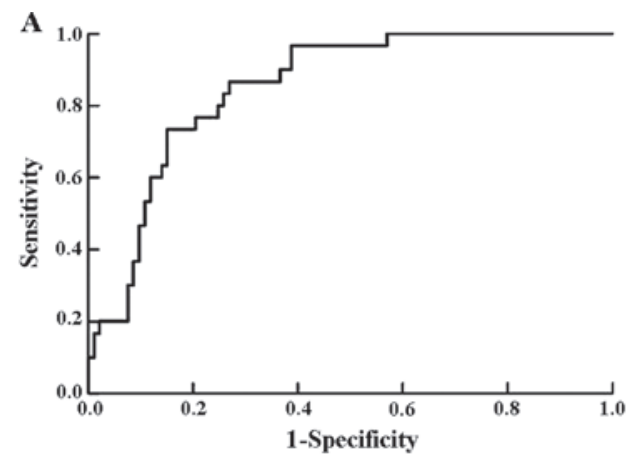

C

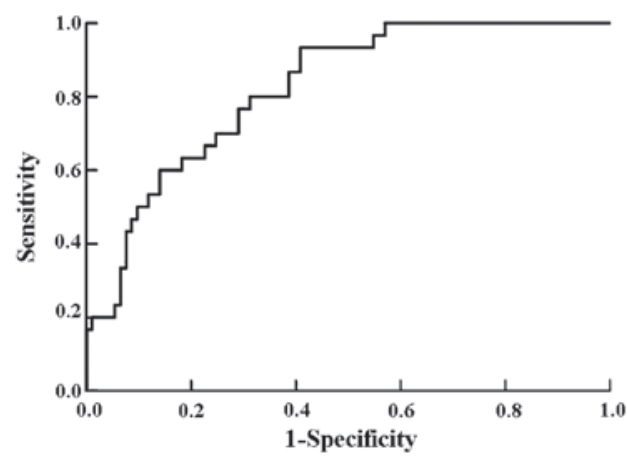

artery PI. The statistical software package SPSS 18.0 (SPSS, Inc., Chicago, IL, USA) was used for all data analysis.

\section{Results}

A total of 800 pregnant women were recruited for the study. Forty women (5.0\%) did not deliver at our hospital and were lost to follow-up, leaving a study cohort of 760 pregnancies. Thirty-eight women developed preeclampsia, giving an incidence of 5.2\%. There were 100 gestational age-matched normal pregnancies as controls. At the time of blood sampling there was no statistically significant difference in maternal age, body mass index and gestational age, between the women that developed preeclampsia and the normal controls (Table I). However, gestational age at delivery and birth weight were lower in pregnancies that developed preeclampsia compared with the controls.

In pregnancies that developed preeclampsia, the uterine artery PI was increased $(1.61 \pm 0.047$ vs. $1.02 \pm 0.049$, $\mathrm{P}<0.001$; Fig. 1A), as was the level of inhibin A $(1.72 \pm 0.023$ vs. $1.03 \pm 0.063, \mathrm{P}<0.001$; Fig. $1 \mathrm{~B})$ and the level of activin $\mathrm{A}$ as compared with the controls $(1.68 \pm 0.38$ vs. $1.06 \pm 0.42$, $\mathrm{P}<0.001$; Fig. 1C). In contrast, the level of PlGF was decreased in pregnancies that developed preeclampsia compared with the controls $(0.69 \pm 0.23$ vs. $1.00 \pm 0.26, \mathrm{P}<0.001$; Fig. 1D). Fig. 2 shows the ROC curves for each marker.

The ROC curves for combinations of the markers in the prediction of preeclampsia are shown in Fig. 3. Combining activin $\mathrm{A}$, inhibin $\mathrm{A}$ and uterine artery PI using logistic regression analysis yielded an area under the curve (AUC) of 0.917 [95\% confidence interval (CI), 0.820-0.918; $\mathrm{P}<0.001]$

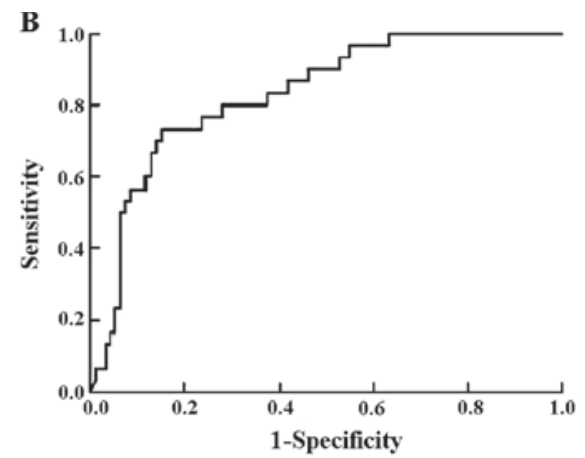

D

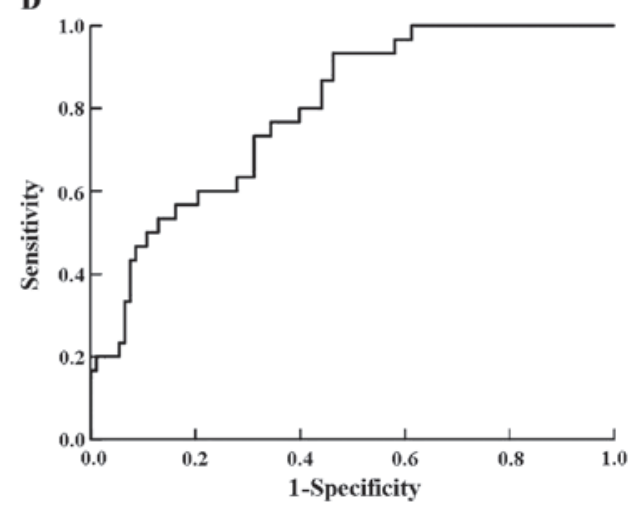

Figure 2. Receiver-operating characteristic (ROC) curves showing the clinical discrimination of uterine artery pulsatility index (PI) and serum markers for predicting preeclampsia. ROC of (A) uterine artery PI, (B) second-trimester inhibin A levels, (C) second-trimester activin A levels and (D) second-trimester placenta growth factor (PlGF) levels for predicting preeclampsia. 


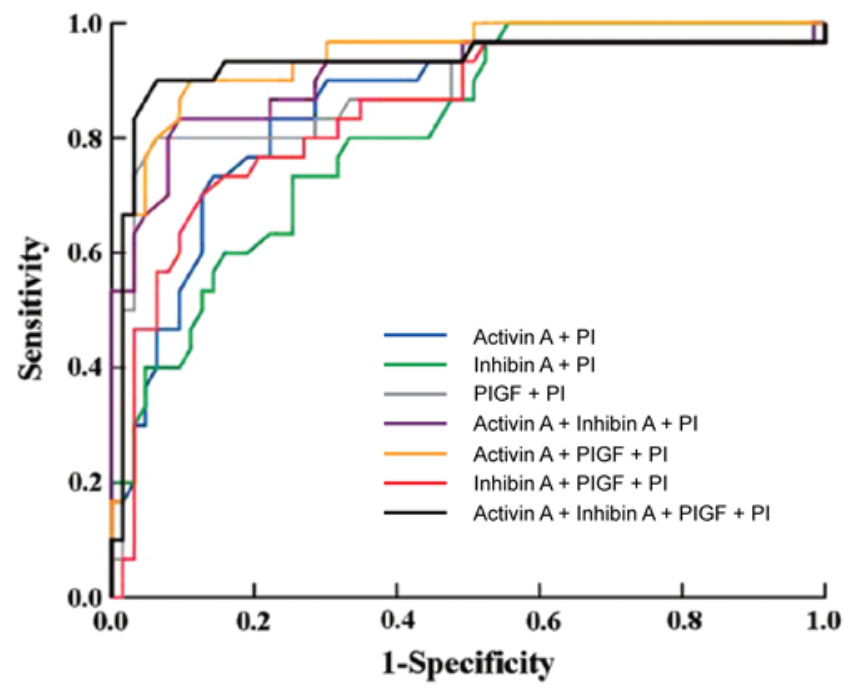

Figure 3. Receiver operating characteristic (ROC) showing the clinical discrimination of different combinations of markers in predicting preeclampsia Activin A plus uterine artery pulsatility index (PI) (blue); inhibin A plus uterine artery PI (green); placenta growth factor (PlGF) plus uterine artery PI (grey); activin A plus inhibin A plus uterine artery PI (purple); activin A plus PIGF plus uterine artery PI (yellow); inhibin A plus PlGF plus uterine artery PI (red); and activin A plus inhibin A plus PIGF plus uterine artery PI (black).

with a sensitivity of $84 \%$ at a specificity of $81 \%$. A combination of activin A, PlGF and uterine artery PI gave an AUC of 0.915 (95\% CI, 0.812-0.928; $\mathrm{P}<0.001$ ) with a sensitivity of $91 \%$ at a specificity of $82 \%$. The combination of all four markers gave an AUC of 0.942 (95\% CI, 0.82-0.991; $\mathrm{P}<0.001)$, with a sensitivity of $92 \%$ at a specificity of $81 \%$.

\section{Discussion}

Preeclampsia is a disorder of pregnancy characterized by high blood pressure and a large amount of protein in the urine $(16,17)$. The disorder usually occurs in the third-trimester of pregnancy and gets worse over time. In severe disease there may be red blood cell breakdown, a low blood platelet count, impaired liver function, kidney dysfunction, swelling, shortness of breath due to fluid in the lungs, or visual disturbances. Preeclampsia increases the risk of poor outcomes for both the mother and the baby. If left untreated, it results in seizures at which point it is known as eclampsia.

There have been many assessments of tests aimed at predicting preeclampsia, although no single biomarker is likely to be sufficiently predictive of the disorder (18-20). Predictive tests that have been assessed include those related to placental perfusion, vascular resistance, kidney dysfunction, endothelial dysfunction, and oxidative stress. A combined screening involving several relevant markers is more likely to provide the best prediction. First-trimester screening would represent a major advantage over a second-trimester approach since it opens prospects for early and more efficient interventions. The aim of this study was to evaluate, in a group of nulliparous women, whether the measurement of maternal serum inhibin A, activin $\mathrm{A}$ and PlGF at 3-4 months' gestation or a combination of these biochemical markers with the second-trimester uterine artery PI are useful in predicting preeclampsia.
Uterine artery PI is an important marker for the prediction of preeclampsia. In our study, the sensitivity was $76 \%$ with the specificity set at $80 \%$ and it dropped slightly to $57 \%$ for a specificity of $90 \%$. Consistent with the literature, we showed an increased uterine artery PI during the late second-trimester in patients who developed preeclampsia (14,21-25).

The clinical manifestations of preeclampsia are associated with general endothelial dysfunction, including vasoconstriction and end-organ ischemia. Implicit in this generalized endothelial dysfunction may be an imbalance of angiogenic and anti-angiogenic factors (26-28). Both circulating and placental levels of soluble fms-like tyrosine kinase-1 (sFlt-1) are higher in women with preeclampsia than in women with normal pregnancy (29-31). sFlt-1 is an anti-angiogenic protein that antagonizes vascular endothelial growth factor (VEGF) and PIGF (32-34), both of which are pro-angiogenic factors. Soluble endoglin (sEng) has also been shown to be elevated in women with preeclampsia and has anti-angiogenic properties, similar to sFlt-1. Both sFlt-1 and sEng are upregulated in all pregnant women to some extent, supporting the idea that hypertensive disease in pregnancy is a normal pregnancy adaptation gone awry. As natural killer cells are intimately involved in placentation and placentation involves a degree of maternal immune tolerance for a foreign placenta, it is not surprising that the maternal immune system may respond more negatively to the arrival of some placentae under certain circumstances, such as a placenta which is more invasive than normal. Initial maternal rejection of the placental cytotrophoblasts may be the cause of the inadequately remodeled spiral arteries in those cases of preeclampsia associated with shallow implantation, leading to downstream hypoxia and the appearance of maternal symptoms in response to upregulated sFlt-1 and sEng.

Inhibin A has been reported as an early marker in predicting preeclampsia. Roes et al took blood samples at 7-13 weeks' gestation from 90 pregnant women, 30 who later developed preeclampsia and 60 controls, and found that inhibin A was increased 5-fold in the women with preeclampsia. Another study (35) reported a sensitivity of inhibin A in the prediction of preeclampsia of $32 \%$ at a specificity of $90 \%$, concluding that the sensitivity of inhibin A was too low for it to be useful as a marker for predicting preeclampsia, but that, combined with other markers, it could play an important role. Activin A has also been proposed as a marker for predicting preeclampsia. The level was elevated at 10-14 weeks' gestation in women with established preeclampsia (36). In a case-control study, the sensitivity was $82 \%$ at a specificity of $91 \%$. Published data regarding $\mathrm{PlGF}$ in the prediction of preeclampsia are controversial. A sensitivity of $80.4 \%$ at a specificity of $78 \%$ has been reported. A prospective study documented that PIGF was decreased distinctly in early pregnancy in women who subsequently developed preeclampsia, and that the degree of decrease was related to the severity of the disease (37).

In our study, we found that both serum inhibin $\mathrm{A}$ and activin A levels were increased, while the PIGF level was decreased in the early second-trimester in women who developed preeclampsia. It should be borne in mind that the population of our study was entirely ethnic Chinese, and further study is required to determine whether levels of these biochemical markers differ among different ethnic groups. 
Furthermore, while it seems that the prediction of preeclampsia is technically feasible using inhibin A, activin A, PlGF and uterine artery Doppler, the cost-benefit balance of this screening needs to be evaluated. In order to accommodate the numerous scans required to perform uterine artery Doppler, more qualified sonographers would need to be trained.

In conclusion, early second-trimester serum inhibin A, activin A, PIGF and second-trimester uterine artery Doppler PI may add further information for the prediction of preeclampsia. The combination of the three serum markers and uterine artery Doppler PI has the highest prediction value for preeclampsia.

\section{Acknowledgements}

This study was supported by grants from Zhejiang Medical Science and Technology Project.

\section{References}

1. Hodgins S: Pre-eclampsia as underlying cause for perinatal deaths: time for action. Glob Health Sci Pract 3: 525-527, 2015.

2. Lim J, Cloete G, Dunsmuir DT, Payne BA, Scheffer C, von Dadelszen P, Dumont GA and Ansermino JM: Usability and feasibility of PIERS on the move: an mHealth App for pre-eclampsia triage. JMIR Mhealth Uhealth 3: e37, 2015.

3. Chaiworapongsa T, Chaemsaithong P, Yeo L and Romero R: Pre-eclampsia part 1: current understanding of its pathophysiology. Nat Rev Nephrol 10: 466-480, 2014.

4. Cornelius DC and Lamarca B: TH17- and IL-17-mediated autoantibodies and placental oxidative stress play a role in the pathophysiology of pre-eclampsia. Minerva Ginecol 66 243-249, 2014.

5. Craici IM, Wagner SJ, Weissgerber TL, Grande JP and Garovic VD: Advances in the pathophysiology of pre-eclampsia and related podocyte injury. Kidney Int 86: 275-285, 2014.

6. Yu J, Shixia CZ, Wu Y and Duan T: Inhibin A, activin A, placental growth factor and uterine artery Doppler pulsatility index in the prediction of pre-eclampsia. Ultrasound Obstet Gynecol 37: 528-533, 2011.

7. Ghosh SK, Raheja S, Tuli A, Raghunandan C and Agarwal S: Is serum placental growth factor more effective as a biomarker in predicting early onset preeclampsia in early second trimester than in first trimester of pregnancy? Arch Gynecol Obstet 287: 865-873, 2013

8. Weed S, Bastek JA, Anton L, Elovitz MA, Parry S and Srinivas SK: Examining the correlation between placental and serum placenta growth factor in preeclampsia. Am J Obstet Gynecol 207: 140.e1-6, 2012.

9. Woodham PC, Brittain JE, Baker AM, Long DL, Haeri S, Camargo CA Jr, Boggess KA and Stuebe AM: Midgestation maternal serum 25-hydroxyvitamin D level and soluble fms-like tyrosine kinase 1/placental growth factor ratio as predictors of severe preeclampsia. Hypertension 58: 1120-1125, 2011

10. Shokry M, Bedaiwy MA, Fathalla MM, Alsemary A, Elwakil S and Murphy A: Maternal serum placental growth factor and soluble fms-like tyrosine kinase 1 as early predictors of preeclampsia. Acta Obstet Gynecol Scand 89: 143-146, 2010.

11. Lai J, Garcia-Tizon Larroca S, Peeva G, Poon LC, Wright D and Nicolaides KH: Competing risks model in screening for preeclampsia by serum placental growth factor and soluble fms-like tyrosine kinase-1 at 30-33 weeks' gestation. Fetal Diagn Ther 35: 240-248, 2014.

12. Hanita O, Alia NN, Zaleha AM and Nor Azlin MI: Serum soluble FMS-like tyrosine kinase 1 and placental growth factor concentration as predictors of preeclampsia in high risk pregnant women. Malays J Pathol 36: 19-26, 2014.

13. Ghosh SK, Raheja S, Tuli A, Raghunandan C and Agarwal S: Serum placental growth factor as a predictor of early onset preeclampsia in overweight/obese pregnant women. J Am Soc Hypertens 7: 137-148, 2013.
14. Odibo AO, Rada CC, Cahill AG, Goetzinger KR, Tuuli MG, Odibo L, Macones GA and England SK: First-trimester serum soluble fms-like tyrosine kinase-1, free vascular endothelial growth factor, placental growth factor and uterine artery Doppler in preeclampsia. J Perinatol 33: 670-674, 2013.

15. Ghosh SK, Raheja S, Tuli A, Raghunandan C and Agarwal S: Can maternal serum placental growth factor estimation in early second trimester predict the occurrence of early onset preeclampsia and/or early onset intrauterine growth restriction? A prospective cohort study. J Obstet Gynaecol Res 39: 881-890, 2013.

16. Demers S, Bujold E, Arenas E, Castro A and Nicolaides KH: Prediction of recurrent preeclampsia using first-trimester uterine artery Doppler. Am J Perinatol 31: 99-104, 2014.

17. Lai J, Poon LC, Pinas A, Bakalis S and Nicolaides KH: Uterine artery Doppler at 30-33 weeks' gestation in the prediction of preeclampsia. Fetal Diagn Ther 33: 156-163, 2013.

18. Kafkaslı A, Türkçüoğlu I and Turhan U: Maternal, fetal and perinatal characteristics of preeclampsia cases with and without abnormalities in uterine artery Doppler indexes. J Matern Fetal Neonatal Med 26: 936-940, 2013.

19. Zhou A, Dekker GA, Lumbers ER, Lee SY, Thompson SD, McCowan LM and Roberts CT; SCOPE consortium: The association of $A G T R 2$ polymorphisms with preeclampsia and uterine artery bilateral notching is modulated by maternal BMI. Placenta 34: 75-81, 2013.

20. Llurba E, Sánchez O, Domínguez C, Soro G, Goya M, Alijotas-Reig J and Cabero L: Smoking during pregnancy: changes in mid-gestation angiogenic factors in women at risk of developing preeclampsia according to uterine artery Doppler findings. Hypertens Pregnancy 32: 50-59, 2013.

21. Gallo DM, Poon LC, Akolekar R, Syngelaki A and Nicolaides KH: Prediction of preeclampsia by uterine artery Doppler at 20-24 weeks' gestation. Fetal Diagn Ther 34: 241-247, 2013.

22. Takahashi K, Ohkuchi A, Suzuki H, Usui R, Kuwata T, Shirasuna K, Matsubara S and Suzuki M: Biophysical interaction between blood pressure and uterine artery Doppler for the occurrence of early-onset preeclampsia: a prospective cohort study. Pregnancy Hypertens 3: 270-277, 2013.

23. Goetzinger KR, Zhong Y, Cahill AG, Odibo L, Macones GA and Odibo AO: Efficiency of first-trimester uterine artery Doppler, a-disintegrin and metalloprotease 12, pregnancy-associated plasma protein a, and maternal characteristics in the prediction of preeclampsia. J Ultrasound Med 32: 1593-1600, 2013.

24. Weintraub AY, Aricha-Tamir B, Steiner N, Hamou BE, Baron J and Hershkovitz R: Postpartum uterine artery Doppler velocimetry among patients following a delivery complicated with preeclampsia. Hypertens Pregnancy 32: 450-458, 2013.

25. Diguisto C, Le Gouge A, Piver E, Giraudeau B and Perrotin F: Second-trimester uterine artery Doppler, PlGF, sFlt-1, sEndoglin, and lipid-related markers for predicting preeclampsia in a high-risk population. Prenat Diagn 33: 1070-1074, 2013.

26. Lehnen H, Mosblech N, Reineke T, Puchooa A, Menke-Möllers I, Zechner U and Gembruch U: Prenatal clinical assessment of sFlt-1 (soluble fms-like tyrosine kinase-1)/PlGF (placental growth factor) ratio as a diagnostic tool for preeclampsia, pregnancy-induced hypertension, and proteinuria. Geburtshilfe Frauenheilkd 73: 440-445, 2013 (In German).

27. Hassan MF, Rund NM and Salama AH: An elevated maternal plasma soluble fms-like tyrosine kinase-1 to placental growth factor ratio at midtrimester is a useful predictor for preeclampsia. Obstet Gynecol Int 2013: 202346, 2013.

28. Bdolah Y, Elchalal U, Natanson-Yaron S, Yechiam H, Bdolah-Abram T, Greenfield C, Goldman-Wohl D, Milwidsky A, Rana S, Karumanchi SA, et al: Relationship between nulliparity and preeclampsia may be explained by altered circulating soluble fms-like tyrosine kinase 1. Hypertens Pregnancy 33: 250-259, 2014.

29. Rizos D, Eleftheriades M, Karampas G, Rizou M, Haliassos A, Hassiakos D and Vitoratos N: Placental growth factor and soluble fms-like tyrosine kinase- 1 are useful markers for the prediction of preeclampsia but not for small for gestational age neonates: a longitudinal study. Eur J Obstet Gynecol Reprod Biol 171: 225-230, 2013.

30. Ohkuchi A, Hirashima C, Takahashi K, Suzuki H, Matsubara S and Suzuki M: Onset threshold of the plasma levels of soluble fms-like tyrosine kinase 1/placental growth factor ratio for predicting the imminent onset of preeclampsia within 4 weeks after blood sampling at 19-31 weeks of gestation. Hypertens Res 36: 1073-1080, 2013. 
31. Huang QT, Wang SS, Zhang M, Huang LP, Tian JW, Yu YH, Wang ZJ and Zhong M: Advanced oxidation protein products enhances soluble Fms-like tyrosine kinase 1 expression in trophoblasts: a possible link between oxidative stress and preeclampsia. Placenta 34: 949-952, 2013

32. Ben Ali Gannoun M, Bourrelly S, Raguema N, Zitouni H, Nouvellon E, Maleh W, Brahim Chemili A, Elfeleh R, Almawi W, Mahjoub T, et al: Placental growth factor and vascular endothelial growth factor serum levels in Tunisian Arab women with suspected preeclampsia. Cytokine 79: 1-6, 2016.

33. Moore GS, Allshouse AA, Winn VD, Galan HL and Heyborne KD: Baseline placental growth factor levels for the prediction of benefit from early aspirin prophylaxis for preeclampsia prevention. Pregnancy Hypertens 5: 280-286, 2015.

34. Tsiakkas A, Cazacu R, Wright A, Wright D and Nicolaides KH: Maternal serum placental growth factor at 12, 22, 32 and 36 weeks' gestation in screening for pre-eclampsia. Ultrasound Obstet Gynecol 47: 472-477, 2016.
35. Roes EM, Gaytant MA, Thomas CM, Raijmakers MT, Zusterzeel PL, Peters WH and Steegers EA: First trimester inhibin-A concentrations and later development of preeclampsia. Acta Obstet Gynecol Scand 83: 117, 2004.

36. Ong CY, Liao AW, Munim S, Spencer K and Nicolaides KH: First-trimester maternal serum activin A in pre-eclampsia and fetal growth restriction. J Matern Fetal Neonatal Med 15: 176-180, 2004

37. Taylor RN, Grimwood J, Taylor RS, McMaster MT, Fisher SJ and North RA: Longitudinal serum concentrations of placental growth factor: evidence for abnormal placental angiogenesis in pathologic pregnancies. Am J Obstet Gynecol 188: 177-182, 2003. 